\title{
Autophagy is required for toll-like receptor-mediated interleukin-8 production in intestinal epithelial cells
}

\author{
YONG-YU LI ${ }^{1}$, SHUNJI ISHIHARA ${ }^{1}$, M. MONOWAR AZIZ ${ }^{1,2}$, AKIHIKO OKA $^{1}$, \\ RYUSAKU KUSUNOKI $^{1}$, YASUMASA TADA ${ }^{1}$, TAKAFUMI YUKI ${ }^{3}$, YUJI AMANO ${ }^{3}$, \\ MESBAH UDDIN ANSARY ${ }^{1}$ and YOSHIKAZU KINOSHITA ${ }^{1}$
}

\author{
${ }^{1}$ Department of Internal Medicine II, Shimane University School of Medicine, Izumo, Shimane, Japan; \\ ${ }^{2}$ Department of Surgical Research, The Feinstein Institute for Medical Research, North Shore University \\ Hospital and Long-Island Jewish Medical Center, Manhasset, NY 11030, USA; ${ }^{3}$ Department of \\ Gastrointestinal Endoscopy, Shimane University Hospital, Izumo, Shimane, Japan
}

Received October 11, 2010; Accepted November 22, 2010

DOI: $10.3892 / \mathrm{ijmm} .2011 .596$

\begin{abstract}
Autophagy is an evolutionarily conserved process that maintains cellular homeostasis via synthesis, degradation, and subsequent recycling of cellular products under various physiological conditions. However, the link between autophagy and the innate immune system remains unknown. In the present study, we evaluated Toll-like receptor (TLR)mediated autophagy induction in intestinal epithelial cells (IECs) and its relationship to interleukin (IL)-8 production. IEC-6, HCT-15, RAW264.7, and THP-1 cells were cultured with or without various TLR ligands, followed by evaluation of the expressions of pro-inflammatory cytokines [IL-8, cytokine-induced neutrophil chemoattractants (CINC)-2 $\beta$, macrophage inflammatory protein (MIP)-2] by real-time PCR and ELISA. To reveal the status of autophagy in IECs and macrophages, light chain 3 (LC3)-II expression was examined using Western blotting and immunofluorescence with confocal microscopy. Also, to evaluate the influence of TLR ligands on autophagy-mediated innate-immune responses, autophagy-related gene (Atg) 7 specific siRNA was transfected into intestinal epithelial cells and IL-8 expression was determined following exposure to various TLR ligands. Cells treated with the TLR ligands produced considerable amounts of pro-inflammatory cytokines (IL-8, CINC-2 $\beta$, MIP-2). Furthermore, the basal levels of LC3-II were markedly higher in IECs as compared to those in macrophages. Our findings indicated that autophagy induction following TLR ligand stimulation was not significantly evident in IECs as compared to macrophages. In addition, Atg7 gene
\end{abstract}

Correspondence to: Dr Shunji Ishihara, Department of Internal Medicine II, Shimane University, Faculty of Medicine, 89-1 Enya-cho, Izumo, Shimane 693-8500, Japan

E-mail: si360405@med.shimane-u.ac.jp

Key words: autophagy, Toll-like receptor, light chain 3, interleukin-8, intestinal epithelial cells expression silencingled to down-regulation of TLR-mediated IL-8 expression in IECs, which indicates a potential role of autophagy in generating innate-immune responses. In conclusion, autophagy may be an important intracellular machinery for inducing the innate immune system in IECs.

\section{Introduction}

In mammalian systems, autophagy, a process that degrades cell components through the lysosomal machinery, thus helping to maintain a balance between synthesis, degradation, and subsequent recycling of cellular products is evolutionarily conserved (1). The process of autophagy is characterized by formation of double-membrane vesicles known as autophagosomes around a targeted region of the cell, mediated by the autophagy-related gene (Atg)12-Atg5-Atg16 complex and by microtubule-associated protein light chain 3 (LC3)phospholipid conjugates (LC3-II) $(2,3)$. The resultant vesicle then fuses with a lysosome and subsequently degrades the cellular contents $(4,5)$. Although autophagy is recognized as a homeostatic process that enables eukaryotic cells to survive during starvation, recent studies have also revealed a variety of roles for autophagy in the regulation of cell death, differentiation, and anti-microbial responses (6-8).

Innate immunity is triggered by pattern recognition receptors (PRRs) that sense pathogen-associated molecular patterns (PAMPs), including lipopolysaccharide (LPS), flagellin, peptidoglycans, and bacterial DNA $(9,10)$. The Toll-like receptor (TLR) family, an important class of PRRs, is well known to induce expression of various inflammatory genes in response to microbial components, which regulates the balance between activation and inhibition of the innate immune system (11). TLRs play essential roles in gut immunity, barrier function and healing during intestinal inflammation (12-16). In particular, the monolayer of intestinal epithelial cells (IECs) is endowed with a capacity for first-line defense against microbial pathogens, which contributes to the regulation of the gut innate immunity under physiological and pathological conditions (17-20). 
The role of autophagy in the innate immunity has been emphasized in several recent publications. The link between autophagy and the innate immune system was shown by the discovery that intracellular pathogens can be eliminated from cells via a TLR-induced autophagy pathway, which may help to maintain normal homeostasis during pathogen infection (21-24). Also, genome-wide association studies recently indicated that autophagy is an essential factor in a variety of disease states including inflammatory bowel disease (IBD) $(25,26)$. Suppression of autophagy can lead to inflammation and tissue damage resembling Crohn's disease (CD), consistent with the identification of allelic loss of Atg16L1 and immunity-related GTPase M (IRGM) as risk factors of CD development (27-29). Although TLR-dependent induction of autophagy has been noted in numerous studies (21-24), the role of autophagy in the gut innate immune system remains largely unknown. To understand the pathogenesis of innate immune-related gut disorders, it is considered important to clarify the crosstalk that occurs between the TLR-mediated pathway and autophagy in IECs, which respond directly to luminal microbial components.

In the present study, we evaluated TLR-mediated autophagy induction in IECs and compared it to that in macrophages, as well as its relationship to the production of interleukin (IL)-8. Our results indicate that IECs have a high basal level of autophagy even without stimulation by various TLR ligands, while we were interested to note that expression of the autophagy system was not altered when stimulated with those TLR ligands. In addition, we also revealed that a deficiency of the autophagy pathway caused by transfection with Atg7 siRNA significantly decreased TLR-mediated IL-8 production in IECs. These are the first known results to show that autophagy may be an essential system for regulation of TLR-mediated IL-8 production in IECs.

\section{Materials and methods}

Reagents. The following reagents and antibodies (Abs) were used in our experiments: purified flagellin from S. typhimurium (InvivoGen, CA, USA), purified LPS, E. coli LPS (InvivoGen), purified Pam2CSK4 (InvivoGen), rapamycin (Sigma, St. Louis, MO, USA), Lipofectamine ${ }^{\mathrm{TM}}$ RNAiMAX (Invitrogen, CA, USA), a human IL-8 enzyme immune assay (EIA) (Invitrogen), a non-radioactive cell proliferation assay (CellTiter $96^{\circledR}$ AQueous) (Promega, Madison, WI, USA), Atg7 siRNA and control siRNA (Santa Cruz, CA, USA), anti-LC3 Ab (MBL, Nagoya, Japan), anti-p62 Ab (MBL), anti- $\beta$-actin $\mathrm{Ab}$ (Sigma), anti-rabbit IgG (Santa Cruz), FITC-conjugated anti-rabbit IgG (DAKO, Glostrup, Denmark), propidium iodide (Sigma), E64D (Sigma), and pepstatin A (Sigma).

Cell cultures. The human colorectal cancer cell line, HCT-15, the human monocytic leukemia cell line, THP-1, the rat small intestine epithelial cell line, IEC-6, and the mouse macrophage cell line, RAW264.7, were obtained from the American Type Culture Collection (ATCC, Manassas, VA, USA), and grown in RPMI-1640 (Invitrogen) or Dulbecco's modified Eagle's medium (DMEM, Sigma) supplemented with $10 \%$ fetal bovine serum (FBS) (Thermo Scientific, Logan, UT, USA) and penicillin-streptomycin-amphotericin B (Invitrogen). The cell lines were maintained at $37^{\circ} \mathrm{C}$ in $5 \% \mathrm{CO}_{2}$ in a humidified incubator.

$R N A$ extraction and real time-PCR. Total RNA was extracted from each sample using Isogen (Nippon Gene, Japan), equal amounts of RNA were then reverse transcribed into cDNA using a QPCR cDNA kit (Stratagene, CA, USA). All primers (Table I) utilized were flanked by intron-exon junctions using the NCBI BLAST tool and the Primer3 software. Quantitative real-time PCR was performed using a StepOne Real-Time PCR system with SYBR-Green PCR master mix (Applied Biosystems, CA, USA), according to the manufacturer's instructions. The levels of human IL-8, rat cytokine-induced neutrophil chemoattractants (CINC)-2 $\beta$, and mouse macrophage inflammatory protein (MIP)-2 mRNA were normalized to that of $\beta$-actin using a sequence detector software (Applied Biosystems).

Protein extraction and Western blotting. Protein extraction and Western blotting assays were performed as previously described $(30,31)$. Briefly, cells were harvested from cultured dishes and were lysed in RIPA lysis buffer. Protein concentration was determined using the BCA protein assy kit (Thermo Scientific). Total cell lysates ( $20 \mu \mathrm{g}$ ) were separated by Trisglycine SDS-PAGE and then transferred to a polyvinylidene difluoride membrane. After blocking with $10 \%$ skim milk (Difco, Detroit, MI, USA) in PBS (pH 7.4), the membrane was reacted with anti-LC3 (1:1000), anti-p62 (1:1000), or anti- $\beta$-actin Abs (1:5000) at room temperature for $1 \mathrm{~h}$, then reacted with peroxidase-conjugated anti-rabbit $(1: 10000)$ or anti-mouse (1:5000) Abs at room temperature for $1 \mathrm{~h}$. The resulting signals were imaged using an ECL (GE Healthcare, Buckinghamshire, UK). To inhibit degradation of LC3-II protein, E64D and pepstatin A were used for the cultured cells.

Confocal microscopy. After stimulation with TLR ligands for $16 \mathrm{~h}$, cells were washed in PBS and then fixed with $4 \%$ paraformaldehyde (Sigma) for $10 \mathrm{~min}$ at room temperature. Fixed cells were washed with PBS, immersed in $100 \mathrm{mg} / \mathrm{ml}$ of Digitonin for $15 \mathrm{~min}$ at room temperature, washed in PBS, and blocked in blocking buffer (X909; DAKO, Carpinteria, CA, USA) for $1 \mathrm{~h}$ at room temperature. Cells were subsequently incubated with the anti-LC3 antibody (PM036, MBL) for $1 \mathrm{~h}$ at room temperature. After 3 washes in PBS, cells were incubated with FITC-anti-rabbit Ab (DAKO) for $30 \mathrm{~min}$ at room temperature and then washed 3 times in PBS and subsequently incubated with propidium iodide (Sigma). Cells were observed with a Laser Scanning Confocal Microscope (Olympus, FV300), photographed at a x120 magnification, and analyzed using the Olympus confocal microscope software.

Enzyme immune assay (EIA). Proteins extracted from HCT-15 cells and culture supernatants were used for the assays. IL-8 contents were measured using an IL-8 EIA kit, following the manufacturer's protocol. Briefly, appropriate sample amounts were transferred by pipette into appropriate wells of anti-human IL-8-coated microtiter strips, followed by addition of a second biotinylated monoclonal $\mathrm{Ab}$, then incubation was performed at room temperature for $90 \mathrm{~min}$. After removing the excess secondary Ab by washing, the samples 
Table I. Primer sequences.

Gene (Accession no.)

Sequences $\left(5^{\prime}-3^{\prime}\right)$

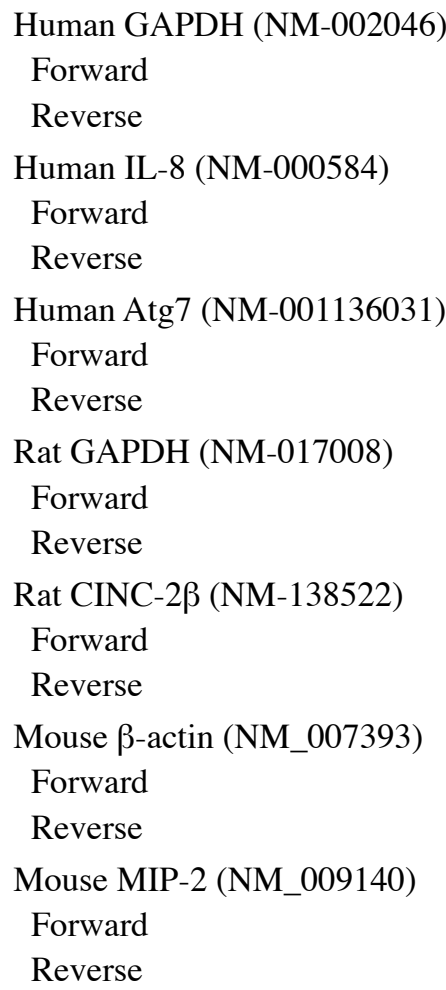

\author{
CCACATCGCTCAGACACCAT \\ TGACCAGGCGCCCAATA
}

TGTGTGTAAACATGACTTCCAAGCT
TTAGCACTCCTTGGCAAAACTG

GATCCGGGGATTTCTTTCACG

CAGCAATGTAAGACCAGTCAAGT

AAGATGGTGAAGGTCGGTGT

GATCTCGCTCCTGGAAGATG

GAGACGGGAATGCAATTTGTTT

GGTCTGCTAGGAATGTTGTCGAT

GATTACTGCTCTGGCTCCTAGC

GACTCATCGTACTCCTGCTTGC

TGTCAATGCCTGAAGACCCTGCC

AACTTTTTGACCGCCCTTGAGAGTGG were incubated with streptavidin-peroxidase, after which a substrate solution was added to produce color that was directly proportional to the concentration of human IL-8 present in the sample. Quantitative results were obtained from a standard curve produced from the experimental findings.

RNA interference. HCT-15 cells were grown in 24-well plates ( $5 \times 10^{4}$ cells/well), then custom siRNAs (Santa Cruz) targeting the human Atg7 gene or control siRNAs were transfected (50 nM/well), according to the manufacturer's protocol. The efficiency of target gene knock-down was assessed by real-time PCR and the results were compared to those of the negative control siRNA-transfected condition. In addition, the efficacy of the Atg7 gene knockdown on autophagy induction was assessed by Western blotting for the detection of LC3-II and p62.

Cell proliferation assay. A non-radioactive cell proliferation assay kit was used to assess cell viability after treatment with Atg7 siRNA. HCT-15 cells were treated with control or Atg7 siRNA, then incubated with TLR ligands for $16 \mathrm{~h}$, after which a cell proliferation assay was performed according to the manufacturer's protocol. The formation of formazan was determined with an EIA plate reader (Bio-Rad, Hercules, CA, USA) at $490 \mathrm{~nm}$ at $4 \mathrm{~h}$ after adding the PMS/MTS solution.

Statistical analysis. All data are expressed as the mean \pm standard error of the mean (SEM). Values were analyzed using the Student's t-test with the SPSS software version 10.1 (San Rafael, CA, USA). ANOVA was used for comparisons of multiple values. P-values $<0.05$ were considered to be significant.

\section{Results}

TLR ligands stimulate production of pro-inflammatory cytokines in cultured IECs and macrophages. Initially, we examined if IECs and macrophages can respond to TLR ligand stimulation (LPS for TLR4, flagellin for TLR5, Pam2CSK4 for TLR2). After $3 \mathrm{~h}$ of stimulation with the ligands, the gene expressions of IL-8, CINC-2 $\beta$ (rat counterpart of human IL-8), and MIP-2 (mouse counterpart of human IL-8) in cultured cells were examined by real-time RT-PCR. In both macrophage cell lines (RAW264.7, THP-1), all ligands tested in this study significantly induced the expressions of MIP-2 and IL-8 (Fig. 1A and B). On the other hand, though stimulation with flagellin and Pam2CSK4 markedly induced CINC-2 $\beta$ in IEC- 6 cells and IL- 8 in HCT-15 cells, stimulation with LPS in these cells resulted in relatively lower levels of the respective cytokines (Fig. 1C and D).

TLR-mediated autophagy induction in IECs and macrophages. The expression level of LC3-II, a mammalian Atg8 homologue widely used as an autophagosome marker and thought to be present on the membranes of autophagosomes, is generally correlated with the number of autophagosomes in a tested sample. In the present study, we assessed LC3-II protein levels in TLR-mediated IECs and macrophages using Western blotting and confocal microscopy. Rapamycin was used as a positive control for autophagy induction. As shown in Fig. 2A, 
A

RAW264.7

MIP-2 mRNA

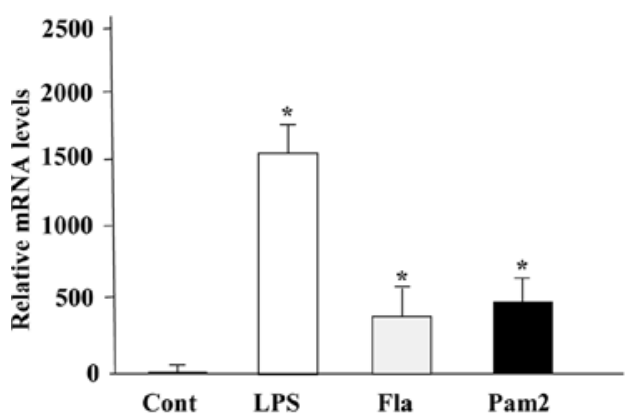

C

IEC-6

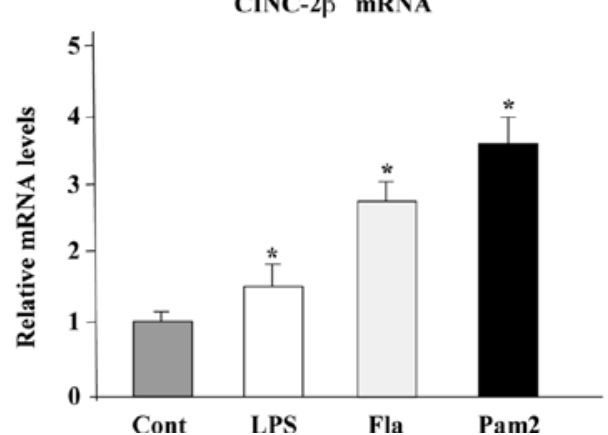

B

THP-1

IL-8 mRNA

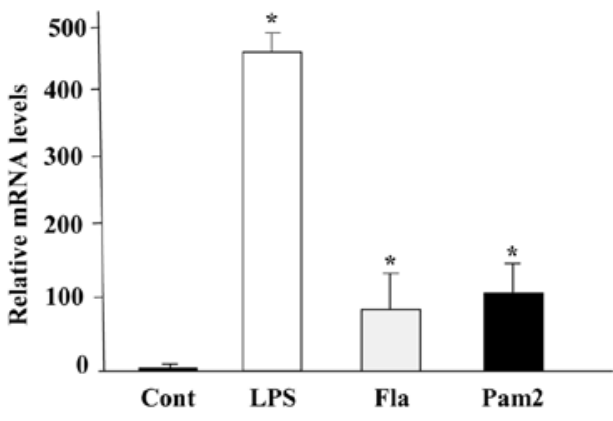

D

HCT-15

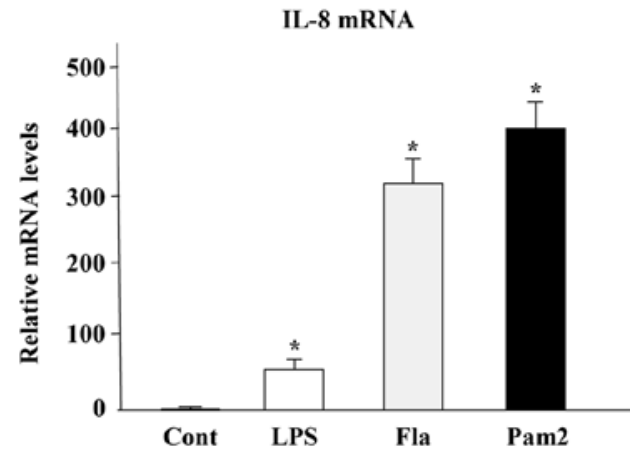

Figure 1. TLR ligand-induced production of pro-inflammatory cytokines in macrophages and IECs. RAW264.7 (A), THP-1 (B), IEC-6 (C), and HCT-15 (D) cells were incubated with various TLR ligands [LPS, $100 \mathrm{ng} / \mathrm{ml}$; flagellin (Fla), $100 \mathrm{ng} / \mathrm{ml}$; Pam2CSK4 (Pam2), $100 \mathrm{ng} / \mathrm{ml}$ ] for $3 \mathrm{~h}$. After ligand stimulation, total RNA was extracted from each sample and the expressions of IL-8, CINC-2 $\beta$ and MIP-2 were examined by real-time RT-PCR. All signals were standardized to the intensity of GAPDH. Error bars indicate the standard error of mean values obtained from 4 independent experiments. ${ }^{*} \mathrm{P}<0.01$ vs. non-stimulated cells.

TLR4 (LPS) and TLR2 (Pam2CSK4) induced abundant levels of LC-3II in macrophages (RAW264.7, THP-1). In contrast, HCT-15 and IEC-6 cells have high basal levels of LC3-II protein in the absence of TLR ligand incubation, which were not changed even following stimulation with the TLR ligands (Fig. 2A). Furthermore, confocal microscopy revealed significant LC3-II immunoreactive signals indicating the presence of autophagosomes in the cytoplasm (green dots). The representative confocal microscopic images shown in Fig. 2B clearly show the results of Western blotting (Fig. 2B), indicating that IECs have a high basal level of LC3-II protein in the absence of TLR ligand incubation.

Effects of Atg7 siRNA on autophagy induction and p62 protein levels in HCT-15 cells. As shown in our experiments, IECs have a high basal level of LC3-II protein even after stimulation with TLR ligands, which was different from the basal autophagy levels observed in macrophages. Based on these findings, we focused on IECs (HCT-15 cells) and investigated the role of autophagy in TLR-stimulated IECs by using a gene knockdown method with an siRNA targeting Atg7. The siRNA used in this study showed a high gene knockdown efficacy, since basal Atg7 gene expression was decreased by $85-90 \%$ (Fig. 3A). The results of Western blotting clearly showed that knockdown of the Atg7 gene decreased LC3-II protein levels in both basal and rapamycin-treated conditions
(Fig. 3B), confirming that RNA interference by Atg7 can inhibit autophagy induction in IECs. In addition, to clearly reveal the siRNA-induced deficiency of autophagy function, we examined p62 levels after treatment with Atg7 or control siRNA by Western blotting. The p62 protein is a selective substrate for autophagy that binds ubiquitin and LC3, which regulates the formation of protein aggregates in the cytoplasm. Once autophagy is induced in cells, p62 protein is immediately degraded. As shown in Fig. 3C, the cellular p62 protein level was increased after treatment with Atg7 siRNA, suggesting that Atg7 siRNA-mediated autophagy deficiency leads to an accumulation of p62 protein in the cytoplasm.

Knockdown of the Atg7 gene does not influence viability of HCT-15 cells in the presence of TLR ligands. After confirming the efficacy of Atg7 siRNA in autophagy induction, we next examined whether siRNA-induced autophagy deficiency influences cell viability before and after stimulation with TLR ligands in serum. The results of our cell proliferation assay clearly show that transfection of Atg7 siRNA does not influence cell viability in the serum-treated condition (Fig. 4A). In addition, treatment with the autophagy inducer rapamycin had no influence on cell viability (Fig. 4B).

Autophagy is required for TLR-mediated IL-8 secretion by HCT-15 cells. After confirming the efficacy of siRNA, the 
A

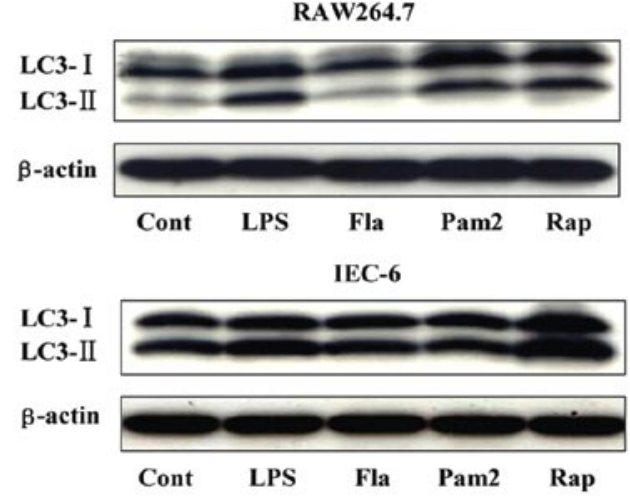

THP-1
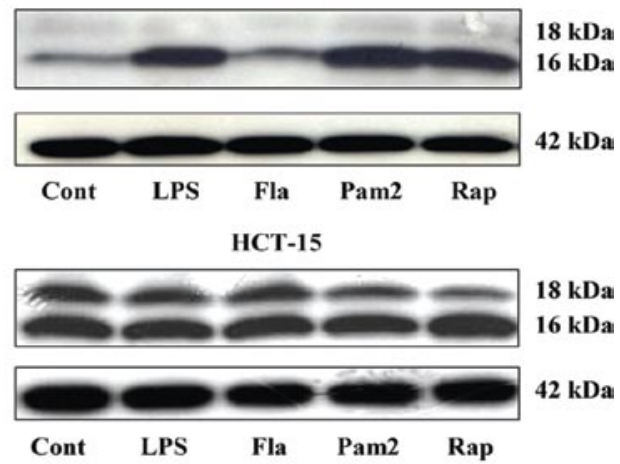

B
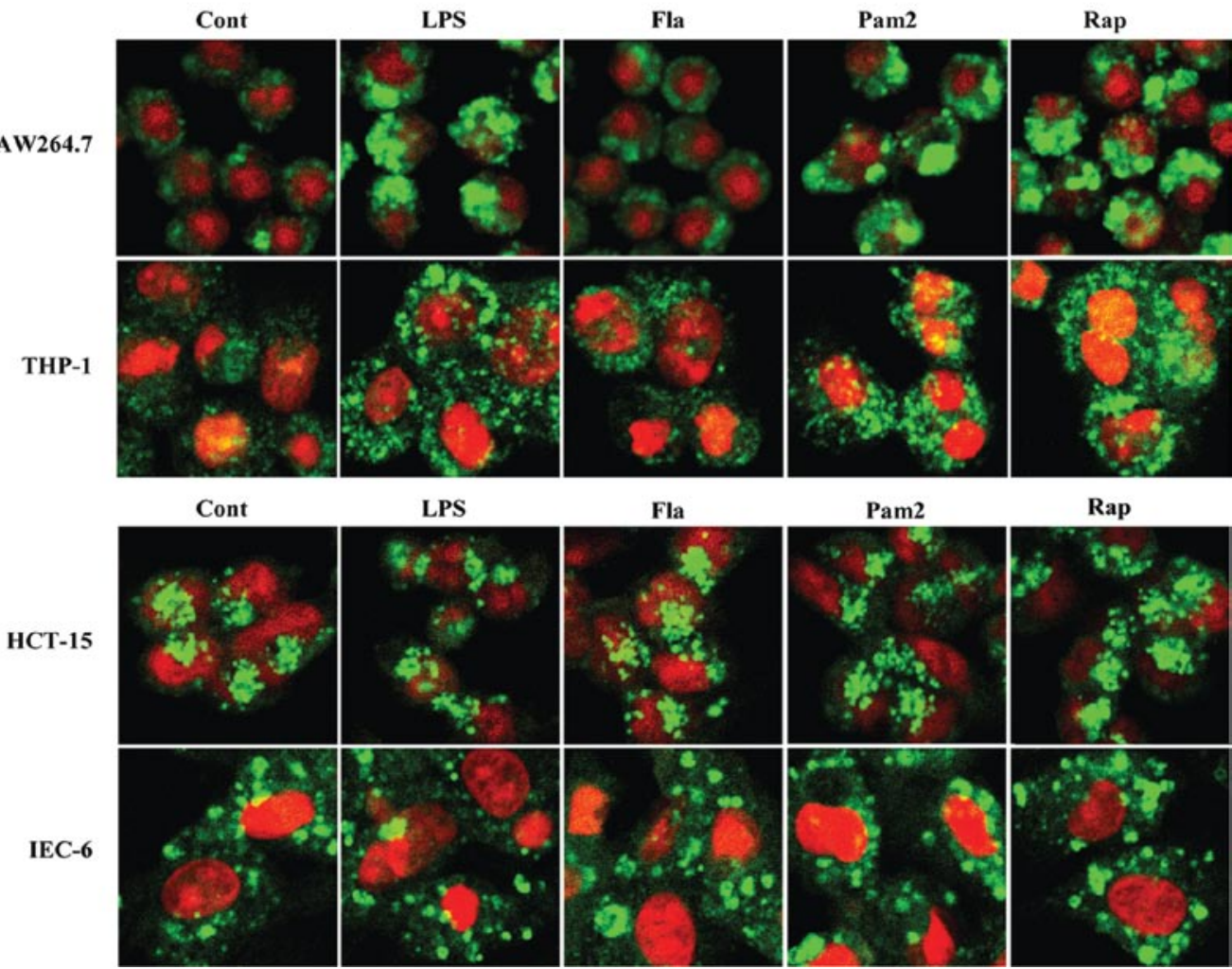

LPS Fla
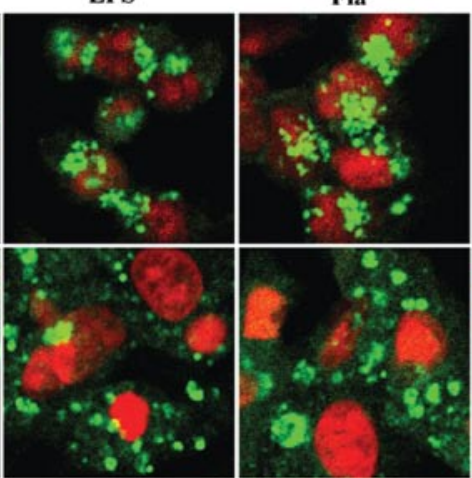

Pam2

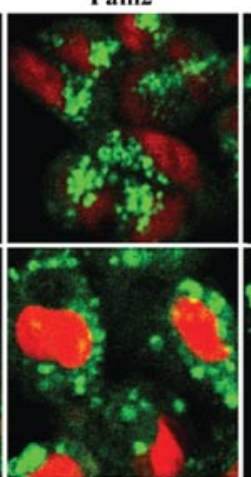

Rap

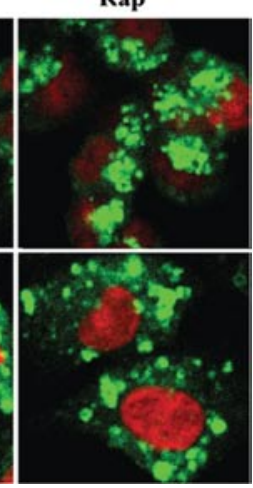

Figure 2. Representative images of TLR-induced autophagy-related protein LC3-II in IECs and macrophages. RAW264.7, THP-1, HCT-15, and IEC-6 cells were incubated with various TLR ligands [LPS, $100 \mathrm{ng} / \mathrm{ml}$; flagellin (Fla), $100 \mathrm{ng} / \mathrm{ml}$; Pam2CSK4 (Pam2), $100 \mathrm{ng} / \mathrm{ml}$ for 16 h. Rapamycin was used as a positive control for autophagy induction. Total LC3-II protein levels and cellular localization were examined by Western blotting (A) and confocal microscopy (B), respectively. Immunoreactive signals (green dots) from LC3-II indicate the presence of autophagosomes in the cytoplasm (B).

role of autophagy in TLR-mediated inflammatory responses in IECs was examined. Control or Atg7 siRNAs were transfected into HCT-15 cells, after which the cells were stimulated with various TLR ligands for $16 \mathrm{~h}$ and the IL-8 contents in the culture supernatants were measured using EIA. As shown in Fig. 5A, TLR ligand-induced IL-8 mRNA expression in Atg7 siRNA-treated cells was significantly lower than that in control siRNA-treated cells. In addition, Atg7 siRNA treatment significantly inhibited TLR-mediated IL-8 protein production (Fig. 5A). To further confirm the role of autophagy, we induced autophagy in IECs by treatment with rapamycin and also examined the TLR-mediated production of IL-8. After stimulation with the TLR ligands, IL-8 mRNA expression and IL-8 contents in the culture supernatants of rapamycin-treated cells were significantly increased as compared to the control cells (Fig. 5B).

\section{Discussion}

In the present study, we found that IECs have a high basal level of autophagy, which did not increase following stimulation with various TLR ligands. In addition, our results clearly demonstrated that Atg7 siRNA-induced inhibition of basal autophagy significantly decreased TLR-mediated IL-8 production in IECs, suggesting that the presence of basal autophagy in IECs is essential for TLR-mediated inflammatory responses to maintain the innate immune system in the gut.

TLRs are sensors of microbial products that initiate host defense responses in various organs (9-12). There is abundant evidence indicating that TLRs play a vital role in maintaining intestinal epithelial homeostasis as well as regulating inflammation, while dysregulation of TLR-mediated signaling leads 
A
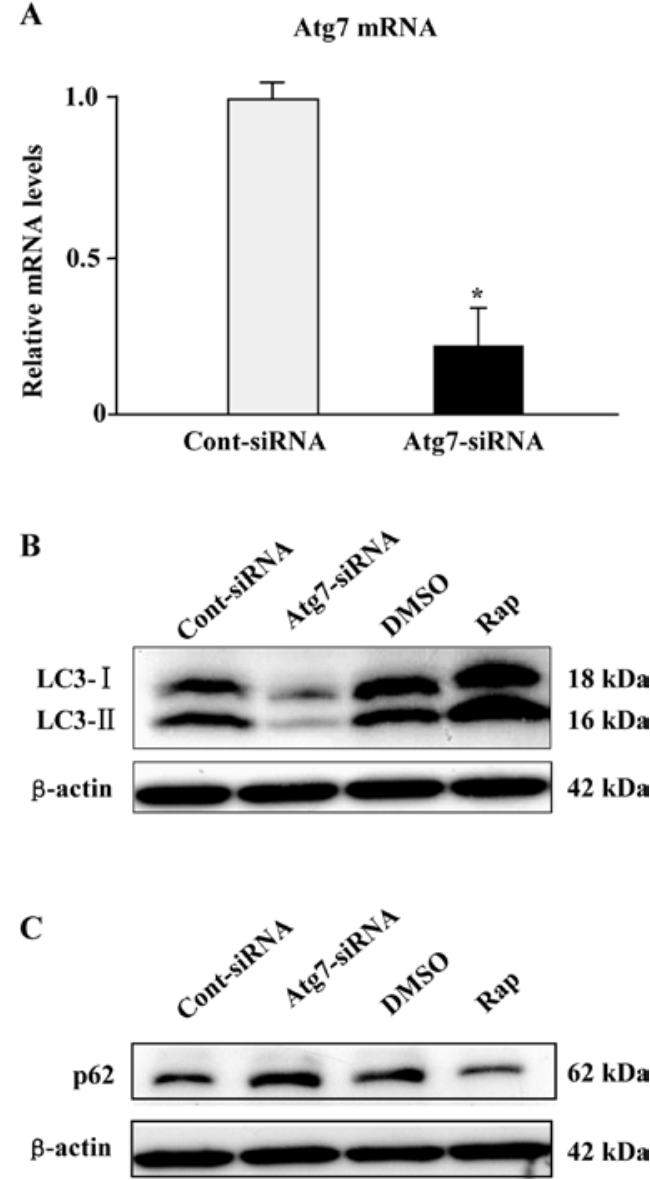

Figure 3. Efficacy of Atg7 siRNA on autophagy induction and p62 protein levels in HCT-15 cells. (A) The efficacy of Atg7 siRNA was evaluated by real-time PCR and compared to that of control siRNA. Error bars indicate the standard error of mean values obtained from 3 independent experiments. ${ }^{*} \mathrm{P}<0.01$ vs. control siRNA. (B) and (C) Representative images of Western blotting showing LC3-II and p62 levels in HCT-15 cells treated with control siRNA, Atg7 siRNA, DMSO (vehicle), and rapamycin.

to development of several gut immune disorders (13-19). Autophagy is also an important physiological process that controls a variety of cellular functions closely associated with the innate immune system (21-24). Although recent findings suggest crosstalk between autophagy and TLR signaling in the intestinal tract, that process remains largely unknown. To investigate this issue, we performed several different in vitro experiments in the present study.

To investigate the effects of stimulation with TLR ligands on autophagy induction, we initially used macrophage cell lines in our in vitro experiments. Stimulation with LPS and Pam2CSK, but not flagellin, significantly increased cellular LC3-II protein levels in RAW264.7 and THP-1 cells, showing that TLR4 and TLR2 are essential sensors for autophagy induction in macrophages. Recently, Xu et al reported that LPS induced autophagy in RAW264.7 cells, which was found to be regulated through a pathway that is dependent on the Toll-IL-1 receptor domain containing adaptor-inducing interferon- $\beta$ (TRIF), while it was independent of the myeloid differentiation factor 88 (MyD88) (32). Delgado et al also demonstrated that activation of TLR4 and TLR7 was associated with autophagy induction in RAW264.7 cells. On the other hand, it has been reported that TLR5 is not a significant
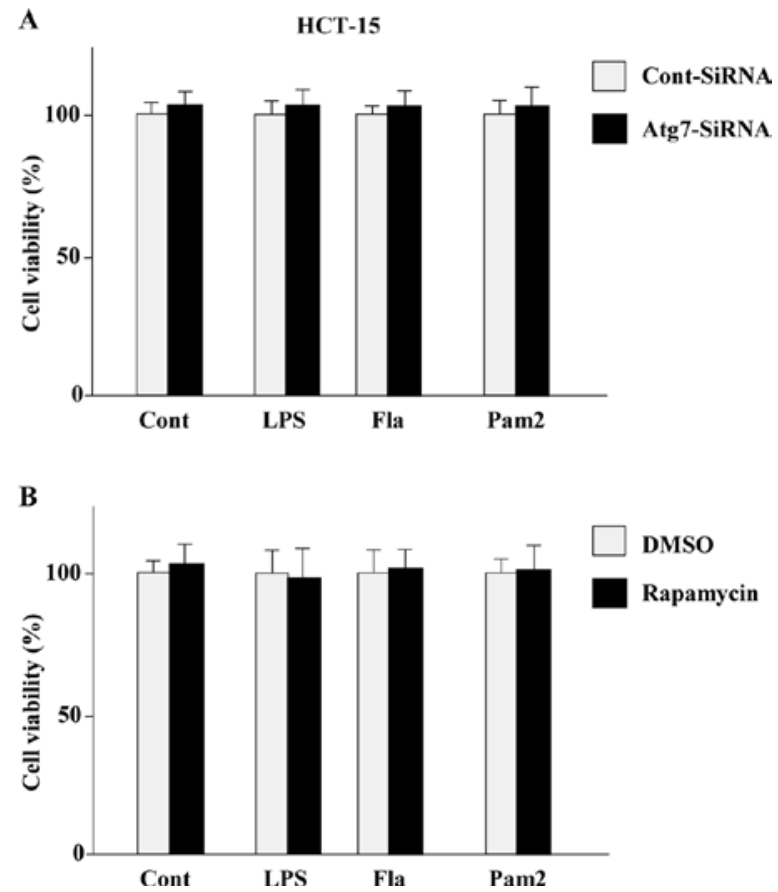

Figure 4. Results of cell proliferation assays. HCT-15 cells were treated with (A) siRNA and (B) rapamycin (Rap), then incubated with various TLR ligands [LPS, $100 \mathrm{ng} / \mathrm{ml}$; flagellin (Fla), $100 \mathrm{ng} / \mathrm{ml}$; Pam2CSK4 (Pam2), $100 \mathrm{ng} / \mathrm{ml}$ for $16 \mathrm{~h}$ and cell viability was assessed. Transfection of Atg7 siRNA did not influence cell viability in the serum-treated condition. Error bars indicate the standard error of mean values obtained from 3 independent experiments.

sensor for autophagy induction in RAW264.7 cells or bone marrow macrophages (23). These recent findings support our results obtained with the macrophage cell lines.

In contrast to those findings, we found that HCT-15 and IEC- 6 cells have high basal levels of autophagy, as determined by the presence of converted LC3-II protein in the cells. Several studies have shown that basal levels of LC3-II protein were detected in cultured IEC lines, including IEC-6, IEC-18, Colon-26, and HT-29 (33-35). Others have also reported that immune-reactive signals of LC3-II were observed in epithelial cells from mice and human intestinal tissue sections. Although various stimuli including serum starvation, glutamine treatment, and $\gamma$-irradiation induce significant autophagy in IECs (33-36), little is known regarding the effect of TLR activation in that process. In the present study, we found that Pam2CSK and flagellin markedly stimulated pro-inflammatory cytokines in HCT-15 and IEC-6 cells. However, these TLR ligands did not have an influence on autophagy induction in these cells. Lee $e t$ al previously indicated an inability to detect induction of autophagy in TLR7-mediated plasmacytoid dendritic cells (pDCs) (37). Furthermore, Schmid et al noted that a high level of constitutive autophagosome formation was observed in DCs, which was not increased after incubation with various immunological agonists (38). Also, a recent study revealed that TLR ligands including LPS did not influence autophagy induction in mice primary cultured macrophages isolated from the peritoneal cavity and bone marrow (27). Together, these findings suggest the possibility that basal autophagy and TLR-mediated autophagy are cell type- and TLR liganddependent. 
A

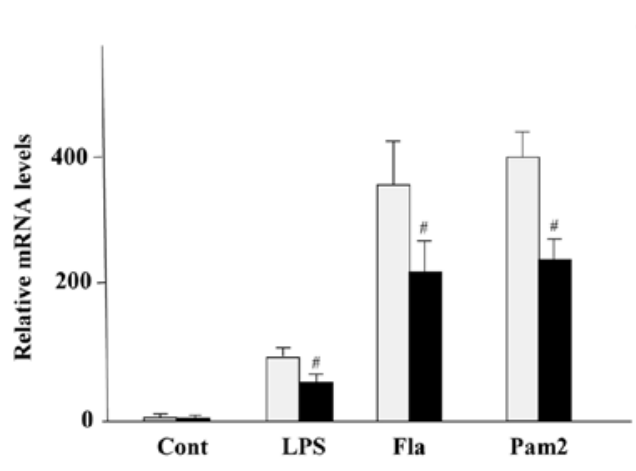

B

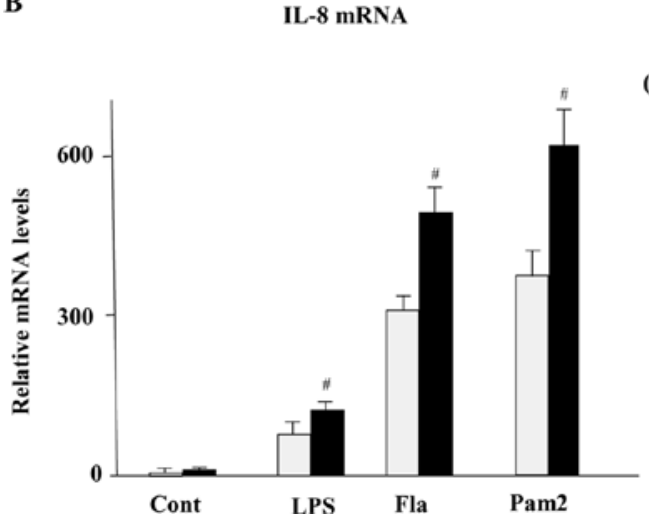

$\begin{array}{ll}\text { IL-8 protein } & \square \text { Cont-siRNA } \\ \text { Atg7-siRNA }\end{array}$

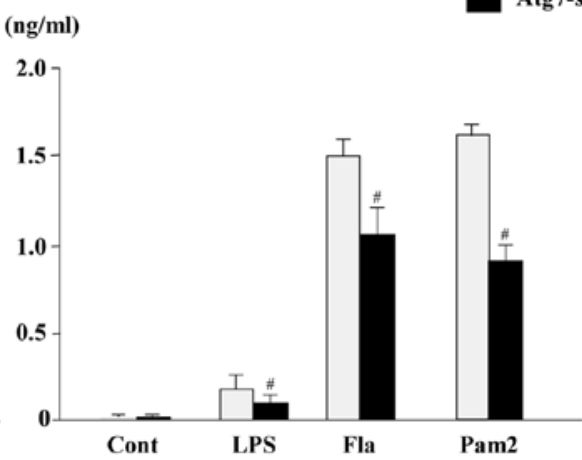

IL-8 protein
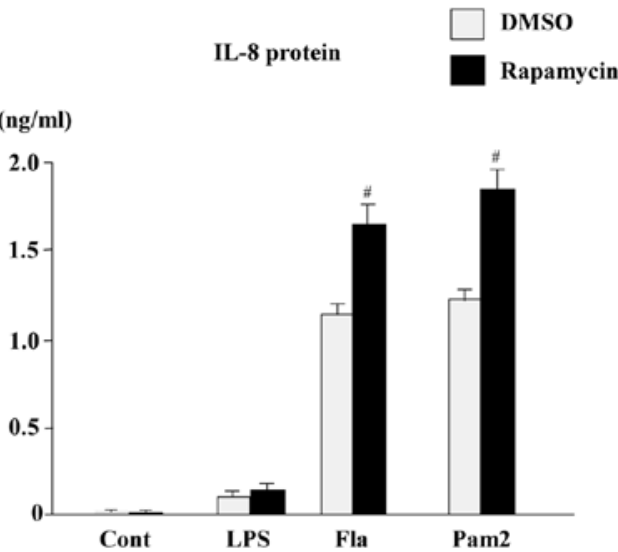

Figure 5. Effects of (A) Atg7 siRNA and (B) rapamycin (Rap) treatment on TLR-mediated IL-8 mRNA expression and IL-8 protein production in HCT-15 cells. HCT-15 cells were treated with siRNA or rapamycin, then incubated with TLR ligands [LPS, $100 \mathrm{ng} / \mathrm{ml}$; flagellin (Fla), $100 \mathrm{ng} / \mathrm{ml}$; Pam2CSK4 (Pam2), $100 \mathrm{ng} / \mathrm{ml}$ ] for $3 \mathrm{~h}$, after which IL-8 mRNA levels were evaluated by real-time PCR. All signals were standardized to the intensity of GAPDH. IL-8 protein contents in the culture supernatants at $16 \mathrm{~h}$ after treatment with TLR ligands were examined by EIA. Error bars indicate the standard error of mean values obtained from 3 independent experiments. ${ }^{*} \mathrm{P}<0.05$ vs. control siRNA or DMSO.

Based on our results, we speculated that high basal levels of autophagy may have certain roles in TLR-mediated inflammatory responses in IECs. Thus, we employed an in vitro system using a gene knockdown method with an siRNA targeting Atg7, an essential gene for autophagy induction. Although gene knockdown of Atg7 did not influence cell viability, TLR ligand-induced IL-8 secretion by Atg7 siRNA-treated cells was significantly lower than that by control siRNA-treated HCT-15 cells. Sasaki et al demonstrated that the secretions of CCL2/monocyte chemotactic protein-1 (MCP-1) and CX3CL1/Fractalkine in cultured biliary epithelial cells induced by various stress factors, such as oxidative stress and serum starvation, were significantly decreased by treatment with the autophagy inhibitor 3-MA (39). Furthermore, a recent study using peripheral blood macrophages clearly revealed that 3-MA or Atg5 siRNA-mediated inhibition of autophagy suppressed influenza virus-induced CXCL10 and interferon- $\alpha$ (IFN- $\alpha)$ (40). These findings support our present results and suggest the possibility that autophagy is required for production of inflammatory cytokines in response to a variety of stimuli, including TLR ligands, in epithelial cells. The mucosal neutrophil response is induced when bacteria stimulate epithelial cells in various organs to secrete several chemokines such as IL-8. Hang et al also found that IL-8 receptor knockout mice have a dysfunctional migration of neutrophils leading to urinary tract infection, which results in tissue destruction (41). Taken together, autophagy may have an essential contribution to maintain innate immune response by regulating inflammatory cytokine production in IECs.

Several studies of crosstalk between autophagy-related genes and intestinal inflammation have been recently reported. Saitoh et al demonstrated that mice lacking Atg16L1 in hematopoietic cells are highly susceptible to dextran sulphate sodium (DSS)-induced experimental acute colitis (27). They also found enhanced levels of LPS-induced IL-1 $\beta$ production in macrophages isolated from this mice strain, indicating that Atg16L1 is an important autophagy-related gene for the inhibition of TLR4-mediated inflammatory response. Apart from the present evidence regarding autophagy functions in macrophages, Cadwell et al found that Atg16L1 or Atg5 mutation leads to disruption of the Paneth cell granule exocytosis pathway, suggesting that abnormal Paneth cell functions may be associated with the pathogenesis of CD (42). Thus, we consider that autophagy has various essential roles in gut immunity, which may be dependent on the cell type and the type of autophagy-regulatory genes.

In summary, we investigated TLR-mediated autophagy induction in IECs, as well as the role of autophagy in IL-8 production in TLR-activated IECs. We have demonstrated that IECs have a high basal level of autophagy, which essentially regulates TLR-mediated IL-8 production in these cells. Our findings provide new insight into crosstalk that occurs 
between autophagy and TLR signaling in IECs. Additional investigations will be necessary to elucidate the precise roles of autophagy in the pathogenesis of intestinal immune disorders.

\section{Acknowledgements}

This study was supported in part by Health and Labour Sciences Research Grants for research on intractable diseases from the Ministry of Health, Labour and Welfare of Japan.

\section{References}

1. Kundu M and Thompson CB: Autophagy: basic principles and relevance to disease. Annu Rev Pathol 3: 427-455, 2008.

2. Mizushima N and Klionsky DJ: Protein turnover via autophagy: implications for metabolism. Annu Rev Nutr 27: 19-40, 2007.

3. Virgin HW and Levine B: Autophagy genes in immunity. Nat Immunol 10: 461-470, 2009.

4. Jung $\mathrm{CH}$, Ro SH, Cao J, et al: mTOR regulation of autophagy. FEBS Lett 584: 1287-1295, 2010.

5. Deretic V: Autophagosome and phagosome. Methods Mol Biol 445: 1-10, 2008.

6. Deretic V: Autophagy in infection. Curr Opin Cell Biol 22: 252-262, 2010

7. Gutierrez MG, Master SS, Singh SB, Taylor GA, Colombo MI and Deretic V: Autophagy is a defense mechanism inhibiting BCG and Mycobacterium tuberculosis survival in infected macrophages. Cell 119: 753-766, 2004.

8. White E, Karp C, Strohecker AM, Guo Y and Mathew R: Role of autophagy in suppression of inflammation and cancer. Curr Opin Cell Biol 22: 212-217, 2010.

9. O'Neill LA, Fitzgerald KA and Bowie AG: The Toll-IL-1 receptor adaptor family grows to five members. Trends Immunol 24: 286-290, 2003.

10. Li X and Qin J: Modulation of Toll-interleukin 1 receptor mediated signaling. J Mol Med 83: 258-266, 2005.

11. Kawai $\mathrm{T}$ and Akira $\mathrm{S}$ : The role of pattern-recognition receptors in innate immunity: update on Toll-like receptors. Nat Immunol 11: 373-384, 2010.

12. Rakoff-Nahoum S, Paglino J, Eslami-Varzaneh F, Edberg S and Medzhitov R: Recognition of commensal microflora by toll-like receptors is required for intestinal homeostasis. Cell 118: 229-241, 2004.

13. Abreu MT, Fukata M and Arditi M: TLR signaling in the gut in health and disease. J Immunol 174: 4453-4460, 2005.

14. Uematsu S and Akira S: Immune responses of TLR5(+) lamina propria dendritic cells in enterobacterial infection. $\mathrm{J}$ Gastroenterol 44: 803-811, 2009.

15. Mayer L: Evolving paradigms in the pathogenesis of IBD. J Gastroenterol: 45: 9-16, 2010.

16. Gribar SC, Anand RJ, Sodhi CP and Hackam DJ: The role of epithelial Toll-like receptor signaling in the pathogenesis of intestinal inflammation. J Leukoc Biol 83: 493-498,2008.

17. Ortega-Cava CF, Ishihara S, Rumi MA, et al: Strategic compartmentalization of Toll-like receptor 4 in the mouse gut. J Immunol 170: 3977-3985, 2003.

18. Ishihara S, Rumi MA, Kadowaki Y et al: Essential role of MD-2 in TLR4-dependent signaling during Helicobacter pyloriassociated gastritis. J Immunol 173: 1406-1416, 2004

19. Oshima N, Ishihara S, Rumi MA, et al: A20 is an early responding negative regulator of Toll-like receptor 5 signalling in intestinal epithelial cells during inflammation. Clin Exp Immunol 159: 185-198, 2010

20. Hansen R, Thomson JM, El-Omar EM and Hold GL: The role of infection in the aetiology of inflammatory bowel disease. $\mathrm{J}$ Gastroenterol 45: 266-276, 2010.
21. Schmid D and Münz C: Innate and adaptive immunity through autophagy. Immunity 27: 11-21, 2007.

22. Delgado MA and Deretic V. Toll-like receptors in control of immunological autophagy. Cell Death Differ 16: 976-983, 2009.

23. Delgado MA, Elmaoued RA, Davis AS, Kyei G and Deretic V: Toll-like receptors control autophagy. EMBO J 27: 1110-1121, 2008.

24. Saitoh T and Akira S: Regulation of innate immune responses by autophagy-related proteins. J Cell Biol 189: 925-935, 2010.

25. Ishihara S, Aziz MM, Yuki T et al: Inflammatory bowel disease: review from the aspect of genetics. J Gastroenterol 44: 1097-1108, 2009.

26. Engel MA and Neurath MF: New pathophysiological insights and modern treatment of IBD. J Gastroenterol 45: 571-583, 2010.

27. Saitoh T, Fujita N, Jang MH, et al: Loss of the autophagy protein Atg16L1 enhances endotoxin-induced IL-1beta production. Nature 456: 264-268, 2008.

28. Kuballa P, Huett A, Rioux JD, Daly MJ and Xavier RJ: Impaired autophagy of an intracellular pathogen induced by a Crohn's disease associated ATG16L1 variant. PLoS One 3: e3391, 2008.

29. McCarroll SA, Huett A, Kuballa P, et al: Deletion polymorphism upstream of IRGM associated with altered IRGM expression and Crohn's disease. Nat Genet 40: 1107-1112, 2008.

30. Aziz MM, Ishihara S, Mishima Y, et al: MFG-E8 attenuates intestinal inflammation in murine experimental colitis by modulating osteopontin-dependent alphavbeta3 integrin signaling. J Immunol 182: 7222-7732, 2009.

31. Aziz MM, Ishihara S, Rumi MA, et al: Prolactin induces MFG-E8 production in macrophages via transcription factor $\mathrm{C} /$ EBPbeta-dependent pathway. Apoptosis 13: 609-620, 2008.

32. Xu Y, Jagannath C, Liu XD, Sharafkhaneh A, Kolodziejska KE and Eissa NT: Toll-like receptor 4 is a sensor for autophagy associated with innate immunity. Immunity 27: 135-144, 2007.

33. Maynard AA, Dvorak K, Khailova L, et al: Epidermal growth factor reduces autophagy in intestinal epithelium and in the rat model of necrotizing enterocolitis. Am J Physiol Gastrointest Liver Physiol 299: G614-G622, 2010.

34. Sakiyama T, Musch MW, Ropeleski MJ, et al: Glutamine increases autophagy under Basal and stressed conditions in intestinal epithelial cells. Gastroenterology 136: 924-932, 2009.

35. Li J, Hou N, Faried A, Tsutsumi S, Takeuchi T and Kuwano H: Inhibition of autophagy by 3-MA enhances the effect of 5-FUinduced apoptosis in colon cancer cells. Ann Surg Oncol 16: 761-771, 2009.

36. Gorbunov NV and Kiang JG: Up-regulation of autophagy in small intestine Paneth cells in response to total-body gammairradiation. J Pathol 219: 242-252, 2009.

37. Lee HK, Lund JM, Ramanathan B, Mizushima N and Iwasaki A: Autophagy-dependent viral recognition by plasmacytoid dendritic cells. Science 315: 1398-1401, 2007.

38. Schmid D, Pypaert M and Münz C: Antigen-loading compartments for major histocompatibility complex class II molecules continuously receive input from autophagosomes. Immunity 26: 79-92, 2007.

39. Sasaki M, Miyakoshi M, Sato Y and Nakanuma Y: Autophagy mediates the process of cellular senescence characterizing bile duct damages in primary biliary cirrhosis. Lab Invest 90 : 835-843, 2010.

40. Law AH, Lee DC, Yuen KY, Peiris M and Lau AS: Cellular response to influenza virus infection: a potential role for autophagy in CXCL10 and interferon-alpha induction. Cell Mol Immunol 7: 263-270, 2010.

41. Hang L, Frendéus B, Godaly G and Svanborg C: Interleukin-8 receptor knockout mice have subepithelial neutrophil entrapment and renal scarring following acute pyelonephritis. J Infect Dis 182: 1738-1748, 2000

42. Cadwell K, Liu JY, Brown SL, et al: A key role for autophagy and the autophagy gene Atg1611 in mouse and human intestinal Paneth cells. Nature 456: 259-63, 2008. 